\title{
A Method for Analyzing the Non-Stationary Nucleation and Overall Transition Kinetics. \\ A Case of Water
}

\author{
Anatolii V. Mokshin'1, a) and Bulat N. Galimzyanov ${ }^{2}$ \\ 1) Kazan Federal University, Kremlevskaya Street 18, 420000 Kazan, \\ Russia \\ ${ }^{2)}$ Kazan Federal University, Kremlevskaya Street 18, 420000 Kazan, \\ Russia
}

(Dated: 28 August 2018)

We present the statistical method as a direct extension of the mean first-passage time concept to the analysis of molecular dynamics simulation data of a phase transformation. According to the method, the mean first-passage time trajectories for the first $(i=1)$ as well as for the subsequent $(i=2,3,4, \ldots)$ nucleation events should be extracted, that allows one to calculate the time-dependent nucleation rate, the critical value of the order parameter (the critical size), the waiting times for the nucleation events and the growth law of the nuclei - i.e. all the terms, which are usually necessary to characterize the overall transition kinetics. There are no restrictions in the application of the method by the specific thermodynamic regions; and the nucleation rate parameters are extracted according to their basic definitions. The method differs from the Wedekind-Bartell scheme and its modification [A.V. Mokshin, B.N. Galimzyanov, J. Phys. Chem. B 116, 11959 (2012)], where the passage-times for the first (largest) nucleus are evaluated only and where the average waiting time for the first nucleation event is accessible instead of the true steady-state nucleation time scale. We demonstrate an efficiency of the method by its application to the analysis of the vapor-to-liquid transition kinetics in water at the different temperatures. The nucleation rate/time characteristics and the droplet growth parameters are computed on the basis of the coarse-grained molecular dynamics simulation data.

Keywords: Nucleation, molecular dynamics, phase transition, water, condensation, growth law, mean first-passage times

a)Electronic mail: anatolii.mokshin@mail.ru 


\section{INTRODUCTION}

The numerical methods of the molecular (particle) dynamics simulations are appearing to be useful tool at the study of the phase transitions $\underline{1}^{-}$. The detailed information about the instantaneous molecular configurations as well as the trajectories of 'molecules', that is accessible due to these simulations, prompts to revise the results of the traditional experiments, and to develop the corresponding theoretical descriptions. Hence, there is a necessity for such the methods, which allows one to compute thermodynamic and kinetic characteristics of the phase transitions within the molecular dynamics (MD) simulation data. Importantly, although the different phase transformations (crystallization, evaporation, condensation etc.) are characterized by quite different physical processes, there are similar regimes in their passing with time ${ }^{2}$. Therefore, the methods developed originally for the computation of the rate characteristics for the some concrete transition (say, crystallization) can be extended with equal success to any first-order phase transition.

One of the most important properties of the phase transition kinetics is the nucleation rate $J(t)$. This quantity defines the number of the overcritical nuclei formed in the motherphase per unit time and unite volume, which are able to grow to the new bulk phase $e^{-\frac{3}{5}}$. Although the nucleation rate is the time-dependent, the some methods adapted specially for its computation within the MD simulations are restricted by the consideration of the steady-state nucleation regime, where $J(t)=J_{s}$. One can mention thereupon the YasuokaMatsumoto method ${ }^{\underline{6}}$, the Volkov method $\underline{7}$ and the method of survival probability $\underline{\underline{8} .9}$, which are aimed for the computation of $J_{s}$. Another scheme used frequently for the analysis of the MD simulation results was suggested in Refs. $\frac{10,11}{1}$ and is known as the Wedekind-Bartell (WB) scheme. It is based on the computation of the average time necessary for the earliest nucleus to reach the different sizes $n$ for the first time, that is, in fact, the mean firstpassage time for the parameter $n$ (see Refs. $\underline{12}, \underline{13}$ ). According to Refs. $\underline{10}, \underline{11}$, the critical size of the nucleus, $n_{c}$, and the steady-state nucleation rate $J_{s}$ can be defined via the resulting mean first-passage time (MFPT) curve generated from the multiple MD simulations.

In this work, we show that the WB-scheme as well as its extended version (see Ref. $\stackrel{14}{ }$ ) do not provide the proper steady-state nucleation rate. Therewith, we present a novel method of statistical analysis, which is based on consideration of the MFPT trajectories defined for the whole succession of the nucleation events. In particular, the method can be applied 
to extract the time-dependent nucleation rate, the average waiting times of the nucleation events, the induction time, the growth laws and, thereby, to quantify the overall transition kinetics. We demonstrate the application of the method to evaluation of the non-stationary droplet nucleation and of the overall vapor-to-liquid transition in water.

\section{DESCRIPTION OF THE METHOD}

Let us assume that the order parameter $n$ characterizes transition of the system from metastable state to stable one, where both states are separated in a free energy landscape $\Delta G(n)$ by a barrier $\Delta G_{n_{c}}$. Although the stochastic features are pronounced in the behavior of the order parameter $n$ during the transition ${ }^{12}$, the time scale $\tau_{s}$ (or by the rate $1 / \tau_{s}$ ) of the barrier crossing could be defined in statistical sense ${ }^{5}$. Practically, the estimation of the term $\tau_{s}$ can be carried out by means of the method of inverted averaging, which is a basis of the MFPT concept ${ }^{13}$. Let us suppose that we are able to observe the temporal evolution of the order parameter $n_{\alpha}(t)$ in an $\alpha$ th experimental run within the ensemble of $M$ experiments, $\alpha \in[1, M]$. From every known trajectory $n_{\alpha}(t)$, one can define the times of the first appearance of the order parameter with the concrete value, i.e. $\tau_{\alpha}(n)$. Then, the mean first-passage times $\bar{\tau}(n)$ comes directly as an average of $\tau_{\alpha}(n)$ over all the experiments,

$$
\bar{\tau}(n)=\frac{1}{M} \sum_{\alpha=1}^{M} \tau_{\alpha}(n) .
$$

The position of the first inflection point in $\bar{\tau}(n)$ corresponds to the critical value $n_{c}$ - that is a location of the free energy barrier $\Delta G_{n_{c}}$. Moreover, the quantity $\bar{\tau}\left(n_{c}\right)$ defines the most probable time scale of the appearance of the critical value $n_{c}$ within the statistics of the $M$ experimental pathways for $n_{\alpha}(t)$. This is a general inference of the MFPT concept $\underline{12}, \underline{13}, \underline{15}$.

This concept can be directly extended to analyzing the nucleation-growth processes. Nucleation is a typical activated process, which represents an initial stage of the phase transition in many-particle system. Moreover, the transition proceeds through the consecutive nucleation events. The following temporal characteristics appears naturally: the waiting time of the first nucleation event $\tau_{1}$ and the nucleation rate $J(t)$. The quantity $\tau_{1}$ defines the time scale necessary to form the first (earliest) nucleus of the critical size ${ }^{16}$ and, thereby, represents a lifetime of the metastable state. Therefore, the quantity $\tau_{1}$ corresponds to a time scale, for which the nucleation rate takes the first detectable nonzero value, i.e. $J\left(\tau_{1}\right)=1 /\left[V(t) \tau_{1}\right]$, 
where $V$ is the volume of a system. Note that the regime of the steady-state nucleation with the rate $J(t)=J_{s}$ can be established in the system after a lapse of the time associated with the so-called induction time $\tau_{\text {ind }} 17$.

Let us associate the order parameter with the nucleus size $n_{\alpha i}(t)$, which is defined as the number of molecules in the nucleus at the time $t$. The term $\alpha$ denotes the index of simulation run, whereas the order number of the nucleation event $i$ indicates that the $i$ th nucleus appears during the $\alpha$ th simulation run. For each $\alpha$ th simulation, we extract the pathway with the $i$ th nucleation event $-n_{\alpha i}(t), i=1,2,3, \ldots-$ at the condition that the configurations for the previous pathways $\left[n_{\alpha j}(t), j=1,2, \ldots,(i-1)\right]$ are ignored. According to Eq. (1), we generate within the extracted data for $\tau_{\alpha i}(n)$ the whole set of

$$
\bar{\tau}_{i}(n)=\frac{1}{M} \sum_{\alpha=1}^{M} \tau_{\alpha i}(n), \quad i=1,2,3, \ldots,
$$

where the quantity $\bar{\tau}_{i}(n)$ at the fixed $i$ represents the mean first-passage times for the size $n$, that is attained by the $i$ th nucleus. Hence, the quantity $\bar{\tau}_{1}(n)$ corresponds to MFPT's for the earliest nucleus, then $\bar{\tau}_{2}(n)$ is the MFPT's for the second nucleus, etc.

What information can be recovered from $\bar{\tau}_{i}(n)$ ?

(i) According to the MFPT concept, the first inflection point on each the curve $\bar{\tau}_{i}(n)$, where the term $\partial \bar{\tau}_{i}(n) / \partial n$ has a maximum, will determine the critical size $n_{c}^{(i)}$ of the $i$ th nucleation pathway. For the moderate degree of metastability of a macroscopic system, it is expected that the critical size is independent on the time, i.e. $n_{c}=n_{c}^{(i)}$. This is one of the basic postulates of the classical nucleation theory $\underline{18}$, which can be violated in the situation of the finite-sized system, for example ${ }^{19-22}$.

(ii) The average waiting time of the $i$ th nucleus with the critical size, which appears in the system, is defined by $\bar{\tau}_{i}\left(n_{c}\right)$. Consequently, the next relation should be fulfilled: $\bar{\tau}_{1}\left(n_{c}\right) \leq \bar{\tau}_{2}\left(n_{c}\right) \leq \bar{\tau}_{3}\left(n_{c}\right) \leq \ldots$, and the quantity $\tau_{1} \equiv \bar{\tau}_{1}\left(n_{c}\right)$ is the average waiting time of the first (earliest) critical nucleus.

(iii) The time-dependent nucleation rate can be calculated directly from the definition as

$$
J(t)=\left.\frac{1}{V(t)} \frac{\partial i(t)}{\partial t}\right|_{t=\bar{\tau}}
$$

where $i$ is the index number of the last nucleation event appearing over the time scale $\bar{\tau}$. The quantity $i(t)$ in Eq. (3) is generated from $\bar{\tau}_{i}\left(n_{c}\right)$ by serial accounting of the critical nuclei, and, thereby, it indicates on the number of the overcritical nuclei at the time $t=\bar{\tau}$. Note 
that Eq. (3) reproduces the most probable scenario for the time-dependent nucleation rate $J(t)$ within the statistics of the $M$ experiments.

(iv) The steady-state nucleation rate $J_{s}$ can be computed for the linear part of the $i(t)$ plots according to Eq. (3). The time scale of the nucleation, which precedes the regime of steady-state nucleation, is characterized by the induction time $\tau_{\text {ind }}$. This quantity is determined as the intersection point of the linear interpolation for $i(t)$ in the regime of the steady-state nucleation,

$$
\frac{i(t)}{V(t)}=J_{s}\left(t-\tau_{i n d}\right),
$$

with the time axis.

(v) The inverted MFPT curve $n_{i}(\bar{\tau})$, [where $\bar{\tau}$ is larger than the time scale of the $i$ th critical nucleus, $\left.\bar{\tau}_{i}\left(n_{c}\right)\right]$ will reproduce statistically the most probable growth law of the $i$ th nucleus. Consequently, such the details of the nuclei growth, like the growth rate and the growth exponent, appear to be available for the $i$ th-order nucleus from the extracted $n_{i}(\bar{\tau})$.

The Wedekind-Bartell scheme. - The adjustment of the MFPT concept to analyze the steady-state nucleation on the basis of the MD simulations had been done and presented in two works 10,11 independently. The authors have shown how the steady-state nucleation rate $J_{s}$, the critical size $n_{c}$, the Zeldovich factor $Z$ can be extracted from the multiple simulations. The MFPT scheme of Refs $\underline{10}, \underline{11}$ - the WB-scheme - suggests to utilize the temporal trajectories of the earliest nucleus in the system and, thereby, it is focused on a single quantity, $\bar{\tau}_{1}(n)$. According to the WB-scheme, the steady-state nucleation rate is defined as

$$
J_{s}^{W B} \propto \frac{1}{2 \bar{\tau}_{1}\left(n_{c}\right)} .
$$

In fact, relation (5) evaluates the reduced inverse average waiting time for the first critical nucleus, but not the actual $J_{s}$. Nevertheless, the WB-scheme can provide the correct values of $J_{s}$. This is possible in a very specific situation, when the number of the critical nuclei increases with time according to

$$
i(t)^{W B}=H\left(t-\tau_{1}\right)\left(\frac{t}{2 \tau_{1}}+\frac{1}{2}\right),
$$

where $\tau_{1} \equiv \bar{\tau}_{1}\left(n_{c}\right)$ and $H(\ldots)$ is the Heaviside step function. It is also necessary to note that the WB-scheme yields the negative induction time $\tau_{\text {ind }}=-\tau_{1}$. 


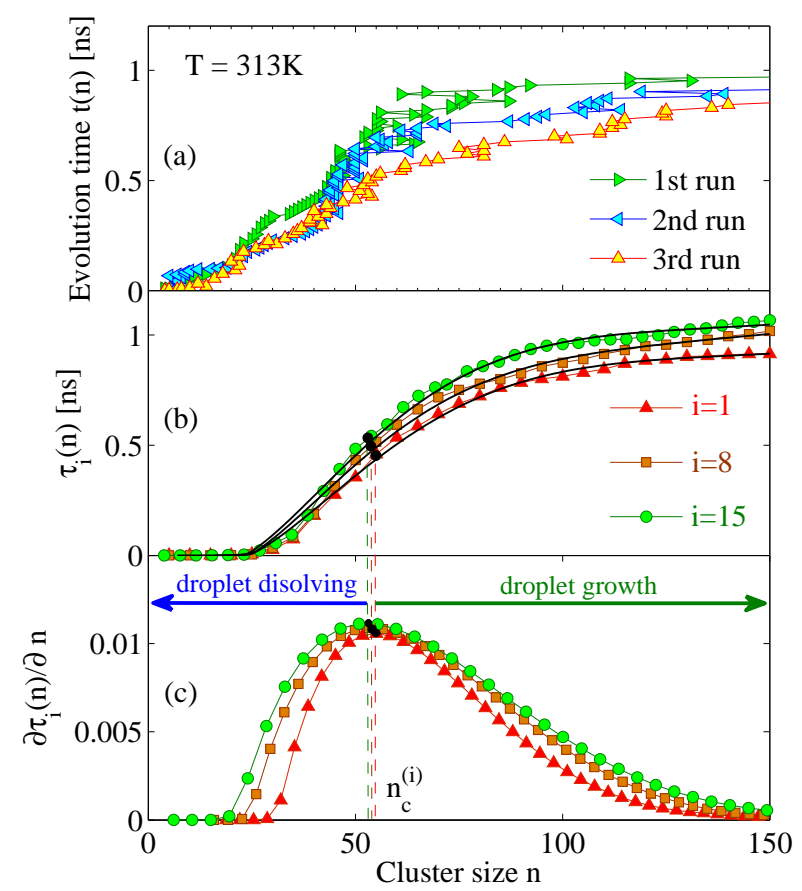

FIG. 1. Analysis of the MD simulation data of water droplet nucleation at the temperature $T=313 \mathrm{~K}$ : (a) Inverted time dependence of the largest droplet size $t\left(n^{(1)}\right)$ as extracted from three independent experiments. (b) MFPT curves for the 1st, 8th and 15th nucleated droplets defined from the set of independent experiments by means of Eq. (2). Trajectories presented in panel (a) were also utilized to define the curve $\bar{\tau}_{1}(n)$. (c) First derivative $\partial \bar{\tau}_{i}(n) / \partial n$ defined for the MFPTcurves given in panel (b), $i=1,8$ and 15 . The position of the maximum on a curve corresponds to the critical size $n_{c}^{(i)}$ of the $i$ th nucleated droplet. The average waiting time of the $i$ th nucleus can be extracted from the MFPT's as $\bar{\tau}_{i}\left(n=n_{c}^{(i)}\right)$.

\section{DROPLET NUCLEATION AND GROWTH IN WATER VAPOR}

In this section we demonstrate the application of the numerical scheme given in Sec. [I] to characterize the droplet nucleation and the subsequent droplet growth in water vapor on the basis of the MD simulation data. These simulation data are obtained within a way identical to given one in Ref. ${ }^{14}$. Here, $N=8000$ water molecules interact in the cubic cell effectively through the anisotropic $\mathrm{mW}$-potential, which represents the Stillinger-Weber potential adopted for water system ${ }^{23}$. Therein, the periodic boundary conditions in all directions were taken. The consideration covers the temperatures from $T=273 \mathrm{~K}$ to $353 \mathrm{~K}$ at the pressure $p \simeq 1$ atm, whilst the $N p T$-ensemble was applied in the particular simulation 
runs. The samples at these temperatures were prepared by means of the isobaric cooling from the well equilibrated vapor configurations.

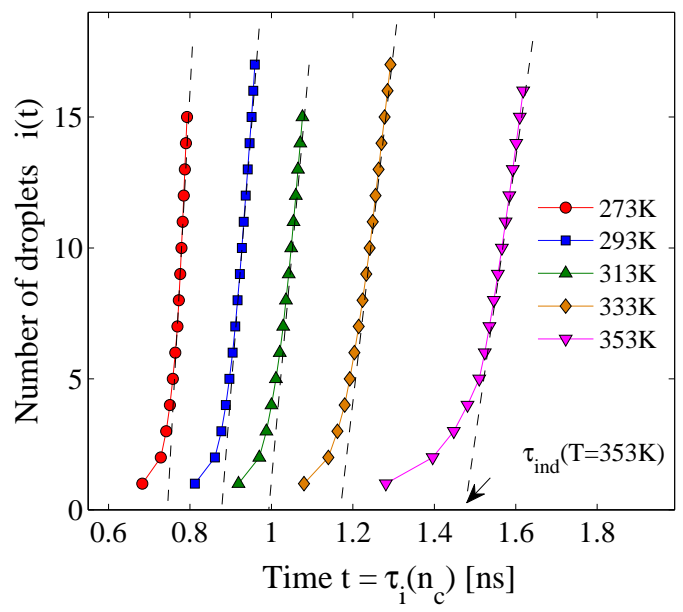

FIG. 2. Isothermic time-dependent number of the nucleated droplets. Each curve is resulted from the generated data for $\bar{\tau}_{i}\left(n_{c}\right)$. Dash lines display the linear interpolation of the data for the steady-state nucleation regime by Eq. (4) with the induction time $\tau_{\text {ind }}$.

TABLE I. Rate characteristics of nucleation-growth kinetics of water droplets at temperature $T$ $(\mathrm{K})$ and vapor number density $\rho_{v}\left(\times 10^{-2} \mathrm{~nm}^{-3}\right)$ : average waiting time of the first droplet $\bar{\tau}_{1}(\mathrm{~ns})$, induction time $\tau_{\text {ind }}(\mathrm{ns})$, steady-sate nucleation rate $J_{s}\left(\times 10^{32} \mathrm{~m}^{-3} \mathrm{~s}^{-1}\right)$ defined according to Eq. (3)), steady-state nucleation rate $J_{s}^{W B}\left(\times 10^{32} \mathrm{~m}^{-3} \mathrm{~s}^{-1}\right)$ from Eq. (5) , steady-state nucleation rate $J_{s}^{M G}$ $\left(\times 10^{32} \mathrm{~m}^{-3} \mathrm{~s}^{-1}\right)$ taken from Ref. $\stackrel{14}{ }$, and growth factor $\mathcal{G}_{c l}\left(\mathrm{~m}^{1 / \nu} \mathrm{s}^{-1}\right)$ with the exponent $\nu=1.3$.

\begin{tabular}{|c|c|c|c|c|c|c|c|}
\hline$T$ & $\rho_{v}$ & $\bar{\tau}_{1}$ & $\tau_{\text {ind }}$ & $J_{s}$ & $J_{s}^{W B}$ & $J_{s}^{M G}$ & $\mathcal{G}_{c l}$ \\
\hline 273 & 1.548 & $0.68 \pm 0.05$ & $0.75 \pm 0.04$ & 84.6 & 0.347 & 0.345 & $576 \pm 60$ \\
\hline 293 & 1.426 & $0.81 \pm 0.07$ & $0.87 \pm 0.05$ & 45.6 & 0.254 & 0.263 & $486 \pm 62$ \\
\hline 313 & 1.353 & $0.92 \pm 0.08$ & $0.99 \pm 0.05$ & 34.1 & 0.210 & 0.211 & $414 \pm 51$ \\
\hline 333 & 1.250 & $1.08 \pm 0.11$ & $1.16 \pm 0.06$ & 23.7 & 0.165 & 0.165 & $357 \pm 55$ \\
\hline 353 & 1.174 & $1.28 \pm 0.13$ & $1.41 \pm 0.06$ & 18.6 & 0.127 & 0.126 & $292 \pm 42$ \\
\hline
\end{tabular}

Thereupon, the samples of water vapor at the desired temperatures $T=[273,293,313$, 333, 353] K were "equilibrated" over a time-scale $\sim 10 \mathrm{ps,} \mathrm{and} \mathrm{the} \mathrm{initial} \mathrm{configurations} \mathrm{for}$ the study of the phase transition kinetics were stored. We generated a hundred independent 
configurations for each $(p, T)$-point to perform the further statistical treatment within the method presented in Sec. II. Identification of the molecules belonging to the vapor or the liquid phase was done using the Stillinger rule $25-27$. As a result, the time-dependent size $n_{\alpha i}(t)$ of each liquid droplet for every simulation run was defined. We remind that the term $\alpha \in[1,100]$ denotes the index of simulation, and $i$ is the order number of the nucleated droplet detected in the $\alpha$ th simulation.

Figure1(a) shows the inverted time-dependent trajectories of the size, $\tau\left(n_{\alpha i}\right)$, of the first $(i=1)$ nucleated droplet as extracted from three different simulations $(\alpha=1,2$ and 3$)$ of the system at the temperature $T=313 \mathrm{~K}$. Averaging over one hundred independent trajectories (these three and others) according to Eq. (21) yields the MFPT curve for the first nucleation event, $\bar{\tau}_{1}(n)$, presented in Fig. 1(b). In addition, Fig. 1(b) demonstrates the MFPT curves computed for the 8th and 15th nucleated droplets. The MFPT curves for other values of the index $i$ have the similar form, and, therefore, are not shown. Following the scheme presented in Sec. III, the derivatives $\partial \bar{\tau}_{i}(n) / \partial n$ were numerically calculated from the MFPT's. As a result, the critical size $n_{c}^{(i)}$ for each $i$ th nucleation event as well as the corresponding nucleation time scale $\bar{\tau}_{i}\left(n_{c}\right)$ were estimated from the positions of the principal maximum in $\partial \bar{\tau}_{i}(n) / \partial n$ [see Fig. 1(c)]. As can be see from Fig. 1(c), the position of the maximum is unchanged with the order of the nucleation event $i$. Consequently, the value of the critical size $n_{c}$ remains unchanged during the nucleation process in the system at the fixed temperature. We found that this is valid for all the considered temperatures. Moreover, the critical size decreases from $n_{c} \simeq 75$ molecules at the temperature $T=273 \mathrm{~K}$ to $n_{c} \simeq 42$ molecules at $T=353 \mathrm{~K}$ (see Ref. $\stackrel{14}{ }$ ).

Figure 2 represents the time dependence of the number of the nucleated water droplets in a system staying at the isothermal-isobaric conditions. Since the time derivative of this term, $\partial i(t) / \partial t$, defines the nucleation rate $J(t, T)$ [see Eq. (3)] , one can see from Fig. 2, that the regime with time-dependent nucleation rate $J(t, T)$ precedes the steady-state nucleation, where the quantity $i(t)$ has a linear time dependence and $J(t, T) \equiv J_{s}(T)$. The extracted values of the induction time are given in Table \. The quantity $\tau_{\text {ind }}(T)$ decreases with falling more deeply into the metastable phase, i.e. with decrease of the temperature $T$, that is in a qualitative agreement with the classical nucleation theory 18 . The driving force of the nucleation grows with the temperature decreasing. Thereby, it accelerates the nucleation and reduces the values of the induction time. Moreover, the observed behavior of $\tau_{\text {ind }}(T)$ 
is correlated with the temperature dependence of the waiting time $\bar{\tau}_{1}(T)$. Namely, we find $\bar{\tau}_{1}(T) / \tau_{\text {ind }}(T)=0.92 \pm 0.01$ for the considered temperature range.

The steady-state nucleation rate $J_{s}(T)$ has been computed according to Eq. (3) as a slope of linear part of each isothermic curve $i(t, T)$ shown in Fig. 2. The extracted values of $J_{s}(T)$ are presented in Table $\mathbb{I}$, where they are compared with $J_{s}^{W B}$ obtained by means of the WB-scheme and with $J_{s}^{M G}$ derived in Ref. 14 through the extended WB-scheme. If the terms $J_{s}^{W B}$ and $J_{s}^{M G}$ have practically the same values, then the both terms underestimate the values of $J_{s}(T)$ by about two orders of magnitude. This difference indicates that the WB-scheme with $J_{s}^{W B}$ as well as the extended WB-scheme with $J_{s}^{M G}$ provides information about the rate of the occurrence of the first (earliest) critical nucleus and does not reproduce the actual steady-state nucleation rate $J_{s}$ of the system. Remarkably, the values of $1 / \bar{\tau}_{1}(T)$ are correlated with for the nucleation rate $J_{s}(T)$, albeit the correlation is not linear.

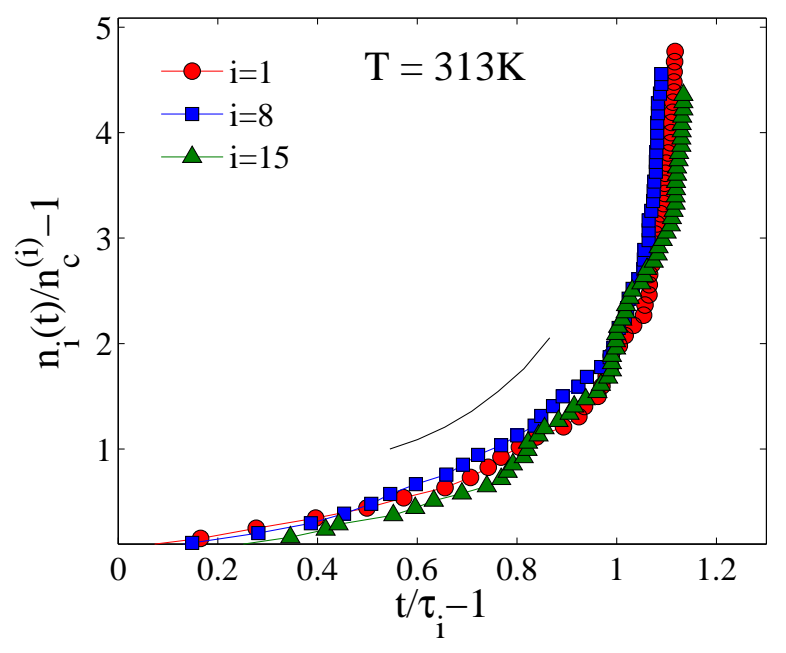

FIG. 3. Rescaled growth curves of the water droplets with the order number $i=1,8$ and 15 . The term $\tau_{i}$ is the waiting time and $n_{c}^{(i)}$ is the critical size of the $i$ th-order nucleus. Note that the data are reproducible by Eq. (8): solid curve shows fragment of the fitting shifted upwards, for clarity.

The consistency of the computed values of $J_{s}$ with the known experimental data $28-32$ can be verified by plotting $\log J_{s}$ versus the scaled quantity $\left(T_{c} / T-1\right)^{3} /\left[\ln \left(p / p_{e q}\right)\right]^{2}$, where a straight line should appear. The resulting graph is known as the scaled Hale plot ${ }^{33,34}$. If the construction of the Hale plot is explicit with the experimental data, then for the treatment of the MD simulation results one needs to know the exact values of the critical temperature $T_{c}$ and the equilibrium vapor pressure $p_{e q}$ as the quantities generated by the 
concrete model of potential. The critical temperature and the saturation curve are not determined for the mW-model; therefore, the corresponding scaled constructions can be speculative ${ }^{35}$. The direct comparison reveals that the values of $J_{s}$ obtained within the $\mathrm{mW}$ model overestimate all the known experimental data by the orders of magnitude, but they approach the result of the MD simulations performed by Yasuoka and Matsumoto with the TIP4P-model: $J_{s}=9.62 \times 10^{32} \mathrm{~m}^{-3} \mathrm{~s}^{-1}$ at $T=350 \mathrm{~K}$ and $\rho_{v}=1.55 \times 10^{-2} \mathrm{~nm}^{-3} \underline{\underline{6} .36}$.

The correctness of the extracted values of the nucleation rate $J(t)$ can be confirmed if these data at the known droplet growth law will reproduce the MD simulation results for the overall condensation kinetics 21 . The growth of the earliest $(i=1)$ droplet for the system can be taken as

$$
R(t)=R_{c}+\left(\mathcal{G}_{c l} t\right)^{\nu}
$$

where $R$ is the droplet radius at the time $t$ elapsed after the appearance of the critical droplet with the radius $R_{c} 37$. The values of the growth factor $\mathcal{G}_{c l}$ are given in Table I; and the growth exponent $\nu=1.3$ appears to be the temperature-independent term. The last equation can be rewritten in the rescaled form for the arbitrary $i$ th-order nucleated droplet:

$$
\frac{n_{i}\left(t, \bar{\tau}_{i}\right)}{n_{c}^{(i)}}=\left[1+\frac{\mathcal{G}_{c l}^{\nu} \bar{\tau}_{i}^{\nu}}{R_{c}^{(i)}}\left(\frac{t}{\bar{\tau}_{i}}-1\right)^{\nu}\right]^{3},
$$

where $n(t)=c_{g} \rho_{c} R(t)^{3}, \rho_{c}$ is the number density of the nucleated phase and $c_{g}$ is the shapefactor, which is $c_{g}=4 \pi / 3$ for a sphere. It is usually accepted by the theoretical treatments that the growth of all the overcritical nuclei $(i=1,2, \ldots)$ in the system follows the same growth law 38,39 . The scheme presented in Sec. II allows one to clarify how the growth of the nuclei emergent at the different times proceeds. Figure 3 presents the rescaled growth curves of the nuclei with the order number $i=1,8$ and 15 for the system at the temperature $T=313 \mathrm{~K}$. Here, each curve is obtained by means of inverted averaging of the independent pathways $n_{\alpha i}(t)$ [see item (v) in Sec. II]. As can be seen, the droplets with the different order numbers ( $i=1,8$ and 15) evolve according to the same growth trajectory, which is reproducible by Eq. (8). Moreover, we found that this result is valid for the droplet growth at other considered temperatures.

The vapor condensation as an overall phase transition is defined by the droplet nucleation and growth. Therefore, the fraction of material condensed into a liquid phase $\phi(t)$ evolves 


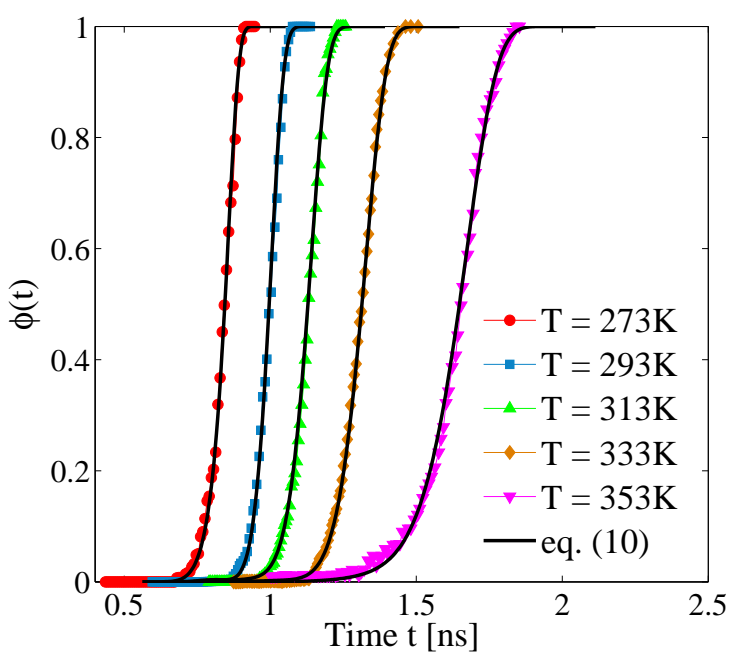

FIG. 4. Time evolution of the condensed liquid fraction in the water vapor at the different temperatures. Markers present results obtained from simulations. Solid curves are predictions of Eq. (10), which contains no adjustable parameters.

with the time according to

$$
\phi(t)=1-\exp \left\{-c_{g} \int_{0}^{t} J\left(t^{\prime}\right)\left[\int_{t^{\prime}}^{t} G\left(t^{\prime \prime}\right) d t^{\prime \prime}\right]^{3} d t^{\prime}\right\}
$$

that is the main equation of the KJMA theory $\underline{\underline{18}}$. Here, $G(t)=d R(t) / d t$ is the instantaneous growth rate of the droplet radius. Taking into account that the critical size is unchangeable with the time and $c_{g} \simeq 4 \pi / 3$, one obtains

$$
\phi(t)=1-\exp \left\{-\frac{4 \pi R_{c}^{3}}{3} \int_{0}^{t} J\left(t^{\prime}\right)\left[1+\frac{\mathcal{G}_{c l}^{\nu}}{R_{c}}\left(t-t^{\prime}\right)^{\nu}\right]^{3} d t^{\prime}\right\} .
$$

Although a single $\alpha$ th experiment produces the inherent trajectory $\phi_{\alpha}(t)$, the most probable scenario of the condensation kinetics $\phi(t)$ can be restored on the basis of the set $\phi_{1}(t), \phi_{2}(t)$, $\ldots, \phi_{\alpha}(t), \ldots, \phi_{M}(t)$ through the inverted averaging [see Eq. (1) ] ? Thus, the fraction $\phi(t)$ is a measurable term. On the other hand, the time-dependent nucleation rate $J(t)$, the growth factor $\mathcal{G}_{c l}$ and the exponent $\nu$ are computed via the scheme presented in Sec. II and, therefore, can be used as input parameters in Eq. (10). As seen from Fig. 目, remarkable agreement is observed between the measured data for $\phi(t)$ and the predictions of Eq. (10). Importantly, the agreement is observed for all the isotherms, and no fitting parameters are utilized within Eq. (10). This is evidence that the computational method presented here 
provides the precise estimates for the nucleation-growth rates. Thereby, it can be used as a convenient tool to study the overall phase transition kinetics.

\section{CONCLUSIONS}

The MFPT approach is known as a pure theoretical concept, which can be applied, for example, for the study of the barrier-crossing problems 12,40 . The application of this approach for analyzing the nucleation kinetics within the MD simulation data had been demonstrated by Wedekind et al. $\underline{\underline{11}}$ and Bartell et al. $\underline{10}$. Recently, the extended version of the approach was reported in Ref. ${ }^{14}$. There are two notable points, which define the applicability of the MFPT approach in such realization. First, the WB-scheme (in a version of Refs $\underline{10}^{11}$ ) is restricted by consideration of the steady-state nucleation, where the nucleation rate $J_{s}$ has no dependence on the time. This corresponds to the distinct temporal regime of the steadystate nucleation within a phase transition passage. Second, the WB-scheme assumes that the waiting time for the first nucleation event, $\bar{\tau}_{1}\left(n_{c}\right)$, and the steady-state nucleation time scale, $\tau_{s}=\left(J_{s} V\right)^{-1}$ are related as $\tau_{s}=2 \bar{\tau}_{1}\left(n_{c}\right)$, that is appropriate for a very specific case of nucleation kinetics. In particular, this condition can be not relevant for the non-equilibrium or driven phase transitions $\stackrel{41}{ }$.

The method presented in this work allows one to define the most probable time scales of the successive nucleation events within a statistics of the independent experiments. Thereby, the nucleation rate can be estimated directly according to its definition as a time-dependent term. Therewith, the critical size and the growth trajectories of the emergent nuclei can be restored with a precision.

In this work, we have analyzed the overall vapor-to-liquid transition kinetics of water within the presented statistical method as applied to the coarse-grained MD simulation data. It is notable that the induction time $\tau_{i n d}(T)$ is correlated with the waiting time for the first nucleation event $\bar{\tau}_{1}(T)$, while both quantities, $\tau_{i n d}(T)$ and $\bar{\tau}_{1}(T)$, exceed the time scale of the steady-state nucleation $\tau_{s}(T)$ more than by two orders in magnitude. In addition, no time( $i$-th order) dependence is detected in the values of the critical size as well as in the character of the droplet growth, that is in agreement with the classical nucleation theory. Finally, we found that the extracted values of the nucleation-growth rates are capable within the KJMA theory to reproduce correctly the MD simulation results for the transformed fraction $\phi(t)$. 


\section{ACKNOWLEDGMENTS}

The authors acknowledge R. M. Khusnutdinoff, Yu. K. Tovbin, G. G. Malenkov, V. V. Brazhkin and V. N. Ryzhov for motivating discussions and their interest to our work. B.N.G. was supported by the Russian Foundation for Basic Research (project No. 12-02-31228).

\section{REFERENCES}

${ }^{1}$ D. Frenkel, B. Smit, Understanding Molecular Simulaton (Academic Press, London, 1996).

${ }^{2}$ J. Frenkel, Kinetic Theory of Liquids (Oxford U.P., London, 1946).

${ }^{3}$ K.F. Kelton, Solid State Physics, ed. by H. Ehrenreich, D. Turnbull (Academic, New York, 1991), Vol.45, p.75.

${ }^{4}$ P. G. Debenedetti, Metastable Liquids: Concepts and Principles (Princeton University Press, Princeton, 1996).

${ }^{5}$ V. P. Skripov, Metastable Liquids (Wiley, New York, 1974).

${ }^{6}$ K. Yasuoka and M. Matsumoto, J. Chem. Phys. 109, 8463 (1998).

${ }^{7}$ I. Volkov, M. Cieplak, J. Koplik, J.R. Banavar, Phys. Rev. E 66, 061401 (2002).

${ }^{8}$ Y. Chushak, L.S. Bartell, J. Phys. Chem. B 105, 11605 (2001).

${ }^{9}$ S.E.M. Lundrigan, I. Saika-Voivod, J. Chem. Phys. 131, 104503 (2009).

${ }^{10}$ L.S. Bartell, D.T. Wu, J. Chem. Phys. 125, 194503 (2006).

${ }^{11}$ J. Wedekind, D. Reguera, and R. Strey, J. Chem. Phys. 126, 134103 (2007).

${ }^{12}$ P. Hänggi, P. Talkner and M. Borkovec, Rev. Mod. Phys. 62, 251 (1990).

${ }^{13}$ S. Redner, A Guide to First-Passage processes (Cambridge University Press, Cambridge, 2001).

${ }^{14}$ A.V. Mokshin, B.N. Galimzyanov, J. Phys. Chem. B 116, 11959 (2012).

${ }^{15}$ A.A. Andronov, E.A. Vitt, and S.E. Khaikin, Theory of Oscillations (Pergamon, Oxford, 1966).

${ }^{16}$ T. Koishi, K. Yasuoka, and T. Ebisuzaki, J. Chem. Phys. 119, 11298 (2003).

${ }^{17}$ V.M. Fokin, N.S. Yuritsyn, and E.D. Zanotto in Nucleation Theory and Applications edited by J.W.P. Schmelzer (Wiley, Weinheim, 2005).

${ }^{18}$ D. Kashchiev, Nucleation: Basic Theory with Appplications (Butterworth-Heinemann, Oxford, 2000). 
${ }^{19}$ A.V. Mokshin, J.L. Barrat, Phys. Rev. E 77, 021505 (2008).

${ }^{20}$ A.V. Mokshin, J.L. Barrat, J. Chem. Phys. 130, 034502 (2009).

${ }^{21}$ A.V. Mokshin, J.-L. Barrat, Phys. Rev. E 82, 021505 (2010).

${ }^{22}$ Yu.K. Tovbin, Russian Journal of Physical Chemistry A 86, 1356 (2012).

${ }^{23}$ V. Molinero, E.B. Moore, J. Phys. Chem. B 113, 4008 (2009).

${ }^{24}$ H. Matsubara, T. Koishi, T.Ebisuzaki, J. Chem. Phys. 127, 214507 (2007).

${ }^{25}$ R.M. Khusnutdinoff, A.V. Mokshin, J. Non-Cryst. Solids 357, 1677 (2011); Physica A 391 , 2842 (2012); A.V. Mokshin, R.M. Yulmetyev, R.M. Khusnutdinov, P. Hänggi, Journal of Experimental and Theoretical Physics, 103, 841 (2006).

${ }^{26}$ V.P. Voloshin, E.A. Zheligovskaya, G.G. Malenkov, and Yu.I. Naberukhin, G. Malenkov, J. Struct. Chem. 42, 794 (2001).

${ }^{27}$ F.H. Stillinger, J. Chem. Phys. 38, 1486 (1963).

${ }^{28}$ R.C. Miller, R.J. Anderson, J.L. Kassner, and D.E. Hagen, J. Chem. Phys. 78, 3204 (1983).

${ }^{29}$ Y. Viisanen, R. Strey, and H. Reiss, J. Chem. Phys. 99, 4680 (1993).

${ }^{30}$ C.H. Heath, K. Streletzky, B.E. Wyslouzil, J. Wölk, and R. Strey, J. Chem. Phys. 117, $6176(2002)$.

${ }^{31}$ A. Khan, C.H. Heath, B.E. Wyslouzil, G. Wilemski, J. Wölk, and R. Strey, J. Chem. Phys. 119, 3138 (2003).

${ }^{32}$ B. Chen, J.I. Siepmann, M.L. Klein, J. Phys. Chem. A 109, 1137 (2005).

${ }^{33}$ B.N. Hale, Phys. Rev. A 33, 4156 (1986).

${ }^{34}$ B.N. Hale, M. Thomason, Phys. Rev. Lett. 105, 046101 (2010).

${ }^{35}$ F. Zipoli, T. Laino, S. Stolz, E. Martin, C. Winkelmann, A. Curioni, J. Chem. Phys. 139, 094501 (2013).

${ }^{36}$ J. Merikanto, H. Vehkamäki, E. Zapadinsky, J. Chem. Phys. 121, 914 (2004).

${ }^{37}$ A.V. Mokshin, G.N. Galimzyanov, J. Phys.: Conf. Series 394, 012023 (2012).

${ }^{38}$ V.A. Shneidman, P. Hänggi, J. Chem. Phys. 101, 1503 (1994).

${ }^{39}$ V.I. Kalikmanov, J. Wölk, T. Kraska, J. Chem. Phys. 128, 124506 (2008).

${ }^{40}$ R. Yvinec, M.R. D’Orsogna, T. Chou, J. Chem. Phys. 137, 244107 (2012).

${ }^{41}$ A.V. Mokshin, B.N. Galimzyanov, J.-L. Barrat, Phys. Rev. E. 87, 062307 (2013). 